\title{
Conformational Relaxation in Glassy State of Poly(methyl mathacrylate)s Investigated by Viscoelasticity and Density Measurements
}

\author{
Osamu Araki, Tsuyoshi Yoshizawa, Toshikazu Takigawa, and Toshiro Masuda ${ }^{\dagger}$ \\ Department of Material Chemistry, Kyoto University, \\ Yoshida, Sakyo-ku, Kyoto 606-8501, Japan
}

(Received May 12, 1999)

\begin{abstract}
Conformational relaxation of poly(methyl methacrylate) in the glassy state has been investigated by measuring dynamic viscoelasticity and density. Physical aging at room temperature was investigated for the samples quenched from $190^{\circ} \mathrm{C}$. The loss tangent $(\tan \delta)$ in the range of temperature below the glass transition temperature decreased with aging time, and approached a certain equilibrium curve from lower temperature side. The rate of change was independent of the molecular weight. However, no effect of physical aging was observed for the samples slowly cooled from $190^{\circ} \mathrm{C}$. The quenched sample and the aged sample after quenching were annealed at 80 or $110^{\circ} \mathrm{C}$ to accelerate the relaxation, and then cooled slowly from each temperature. The temperature dispersion curves of the dynamic viscoelastic functions of the samples agreed well with those of quenched samples in higher temperature range than the annealing temperature, and with those of slowly cooled ones in lower temperature range. Densities measured at $30^{\circ} \mathrm{C}$ were found to explain well the change in segmental conformation due to thermal histories. The effect of thermal history on the viscoelasticity was discussed in terms of the change of segmental conformation of polymeric glasses.

KEY WORDS Conformational Relaxation / Poly(methyl methacrylate) / Polymeric Glass / Dynamic Viscoelasticity / Density / Physical Aging /
\end{abstract}

Physical properties of amorphous polymers in the glassy state are generally affected by the segmental conformation that depends strongly on the thermal history. ${ }^{1-3}$ A glassy polymer sample quenched from a temperature above the glass transition temperature $\left(T_{\mathrm{g}}\right)$ to a temperature below $T_{\mathrm{g}}$ is not in the equilibrium state. When the sample is annealed at a temperature, $T_{\mathrm{A}}\left(<T_{\mathrm{g}}\right)$, the physical properties change toward those in the equilibrium state at $T_{\mathrm{A}}$ by changing the local chain conformation. The behavior is called 'structural relaxation' and/or 'physical aging', and has been investigated by measuring volume recovery, ${ }^{4-9}$ enthalpy recovery, ${ }^{2,6,10,11}$ creep compliance, ${ }^{4,6,11}$ and stress relaxation. ${ }^{12-14}$ Time scales for relaxation of different properties have not been always the same. For example, the approach of volume to the equilibrium is faster than those of enthalpy and creep. ${ }^{8}$ The reason for the different rates has not been well understood, because molecular motion in polymeric glasses is not clear.

Internal structure in glassy polymer is changed and relaxed by the chain conformation change due to the molecular segmental motion. The structural relaxation might be called a conformational relaxation by segmental motion of the polymer chain. Scale of the molecular motion varies with segmental length and the mobility depends on temperature. The motion is generally called a local motion, a segmental motion, a cooperatively rearranging motion $\left(<T_{\mathrm{g}}\right)$ and a reptation $\left(>T_{\mathrm{g}}\right)$ according to the scale of molecular motion.

It has been reported for poly(methyl methacrylate) (PMMA) that the dynamic storage modulus $\left(E^{\prime}\right)$ increases and the dynamic loss modulus $\left(E^{\prime \prime}\right)$ decreases creases and the dynamic loss modulus ( $\left.E^{\prime}\right)$ decreases The values of the viscoelastic functions change, in many cases, linearly with logarithm of the aging time at $T_{\mathrm{A}}{ }^{18}$ The studies on aging for PMMA, however, are mostly limited to the cases at constant temperatures. Little is known about how the dispersion curves of the viscoelastic functions change with aging time. In this study, we measured temperature dispersion curves of dynamic viscoelastic functions of PMMA aged or annealed at $T_{\mathrm{A}}(<$ $T_{\mathrm{g}}$ ) for various time periods to clarify the effect of aging on segmental motion and conformational relaxation of PMMA in a wide temperature range.

\section{EXPERIMENTAL}

\section{Materials}

Two samples of homo-PMMA with different molecular weights were used in this study. Sample code according to average molecular weight in $10^{3}$, molecular weight $\left(M_{w}\right)$ measured by light scattering, molecular weight distribution $\left(M_{w} / M_{n}\right)$ by gel permeation chromatography (GPC), and the glass transition temperature $\left(T_{\mathrm{g}}\right)$ by differential scanning calorimetry (DSC) under a heating rate of $10^{\circ} \mathrm{C} \mathrm{min}{ }^{-1}$ are shown in Table $\mathrm{I}$. The preparation of the film samples was described elsewhere. ${ }^{19}$ Film samples obtained were below $0.1 \mathrm{~mm}$ in thickness. Various thermal histories were applied to the samples. The samples used in this study are summarized in Table II focusing on the thermal histories. The films were heated in a compression molding machine at $190^{\circ} \mathrm{C}$ for $5 \mathrm{~min}$, and then were cooled in two ways: slow cooling from $190^{\circ} \mathrm{C}$ toward room temperature $T_{\mathrm{r}}\left(15-25^{\circ} \mathrm{C}\right)(\mathrm{SC})$ and rapid quenching from 190 to $0^{\circ} \mathrm{C}(\mathrm{QU})$, as described in

Table I. Sample code, molecular characteristics and glass transition temperature $\left(T_{\mathrm{g}}\right)$ measured by DSC at a heating rate of $10^{\circ} \mathrm{C} \mathrm{min}^{-1}$

\begin{tabular}{ccccc}
\hline Sample & $M_{w}$ & $M_{w} / M_{n}$ & $T_{\mathrm{g}} /{ }^{\circ} \mathrm{C}$ & Supplier \\
\hline PMMA575 & 575000 & 2.7 & 123 & Scientific Polymer Products \\
\hline PMMA72 & 72000 & 1.7 & 115 & Mitsubishi Rayon Co. \\
\hline
\end{tabular}

\footnotetext{
${ }_{\dagger}^{\dagger}$ To whom correspondence should be addressed (Tel : +81-75-753-5606, Fax : +81-75-753-4911, e-mail : masuda@rheogate. polym. kyoto-u.ac.jp).
} 
Table II. Various thermal histories ${ }^{\mathrm{a}, \mathrm{b}}$ applied to PMMA samples, $\alpha$-transition temperature $\left(T_{\alpha}\right)$ and density $(\rho)$ at $30^{\circ} \mathrm{C}$ of the samples ${ }^{\mathrm{c}}$

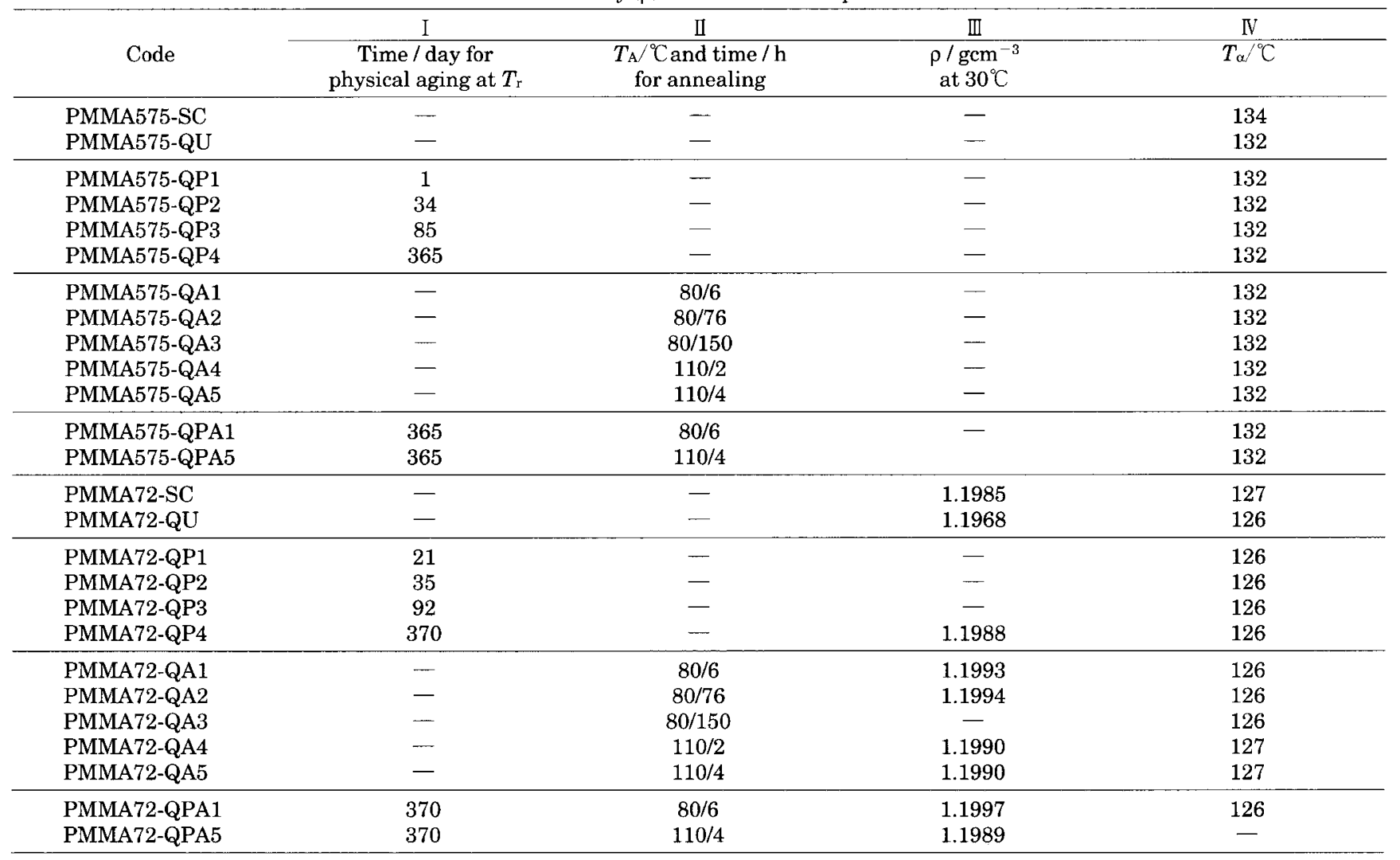

aThermal histories were applied to the samples by two steps. 1st step was slow-cooling or quench from $190^{\circ} \mathrm{C} ; 2$ nd step was physically aging at $T_{\mathrm{r}}$ or annealing at $T_{\mathrm{A}}$. 'The sample codes include the summary of thermal histories. SC stands for slow-cooling; $\mathrm{QU}$ quench; $\mathrm{QP}$ quench and physical aging ; QA quench and annealing; QPA quench, physical aging and annealing. ${ }^{c} T_{x}$ was obtained from the peak temperature of the temperature dispersion curve of $\tan \delta$. Values of $\rho$ were not measured for PMMA575 series.

detail in our previous paper. ${ }^{19}$ Physical aging was made for SC and QU samples at various temperatures below $T_{\mathrm{g}}$, which is regarded as a stage of the thermal history in this study. QU samples were kept at $T_{\mathrm{r}}$ for various days as shown in Column I in Table $\Pi$. The physically aged samples are called QP. Another physical aging was performed at higher temperatures than $T_{\mathrm{r}}$. QU samples were annealed at $T_{\mathrm{A}}\left(T_{\mathrm{A}}=80\right.$ or $\left.110^{\circ} \mathrm{C}\right)$ for several hours in order to accelerate conformational relaxation and then cooled slowly from $T_{\mathrm{A}}$ to $T_{\mathrm{r}}$. The annealed samples thus obtained are called QA. The annealing temperature/time for each sample is shown in Column II of Table II. Moreover, PMMA-QP4 samples were also annealed in the same condition as for QA series. PMMA 575-QP4 samples were annealed at $80^{\circ} \mathrm{C}$ for $6 \mathrm{~h}$ and at $110^{\circ} \mathrm{C}$ for $4 \mathrm{~h}$ and PMMA72-QP4 sample was annealed at $80^{\circ} \mathrm{C}$ for $6 \mathrm{~h}$. The samples are called QPA, samples codes are PMMA575-QPA1, -QPA5, PMMA72-QPA1, and -QPA5 corresponding to annealing condition for QA series.

\section{Measurements}

The temperature dispersion of dynamic viscoelastic functions, $E^{\prime}, E^{\prime \prime}$ and loss tangent $(\tan \delta)$ of the samples was measured at an angular frequency of $1 \mathrm{~s}^{-1}$ and at a static strain of $0.05 \%$ using a Rheometrics RSA- II . For $\mathrm{SC}, \mathrm{QU}$, and QA2 samples, the measurements were also made at an angular frequency of $100 \mathrm{~s}^{-1}$. All measurements were performed on heating at a heating rate of 2
${ }^{\circ} \mathrm{C} \min ^{-1}$. Densities of PMMA72 specimens were measured by a density gradient tube of aqueous sucrose solution at $30^{\circ} \mathrm{C}$ The average density gradient was $2.0 \times 10^{-4}$ $\mathrm{g} \mathrm{cm}^{-3} / \mathrm{cm}$-distance. Accuracy of the value of density was estimated within \pm 0.0001 . The results are tabulated in Column III of Table II .

\section{RESULUTS AND DISCUSSION}

\section{Dynamic Viscoelasticity of PMMA Samples}

The temperature dispersion curves of $E^{\prime}, E^{\prime \prime}$ and $\tan \delta$ of PMMA575-SC, -QU, and -QP4 with three typical thermal histories are shown in Figure 1. The figure clearly demonstrates an effect of physical aging on viscoelastic properties of the samples, as can be seen by comparing $\mathrm{SC}$ and QU samples. The $E^{\prime}$ value of PMMA 575-SC is higher than that of PMMA575-QU at low temperatures (see Zoom). The temperature at onset of the steep decrement of $E^{\prime}$ curve for PMMA575-SC is higher than that of PMMA575-QU. The $E$ " and $\tan \delta$ curves of PMMA575SC clearly exhibit a minimum, while those of PMMA 575-QU do not. At low temperatures, the $E^{\prime \prime}$ and $\tan \delta$ curves of PMMA575-QP4 are located below those of PMMA575-SC, and the $E^{\prime}$ curve of PMMA575-QP4 in slightly higher side than that of PMMA575-SC (see Zoom). The difference in value is small but is not the experimental error, as will be discussed later. At high temperatures, $E^{\prime}, E^{\prime \prime}$ and tan $\delta$ curves of PMMA575-QP4 coincide fairly well with those of PMMA575-QU. The tem- 

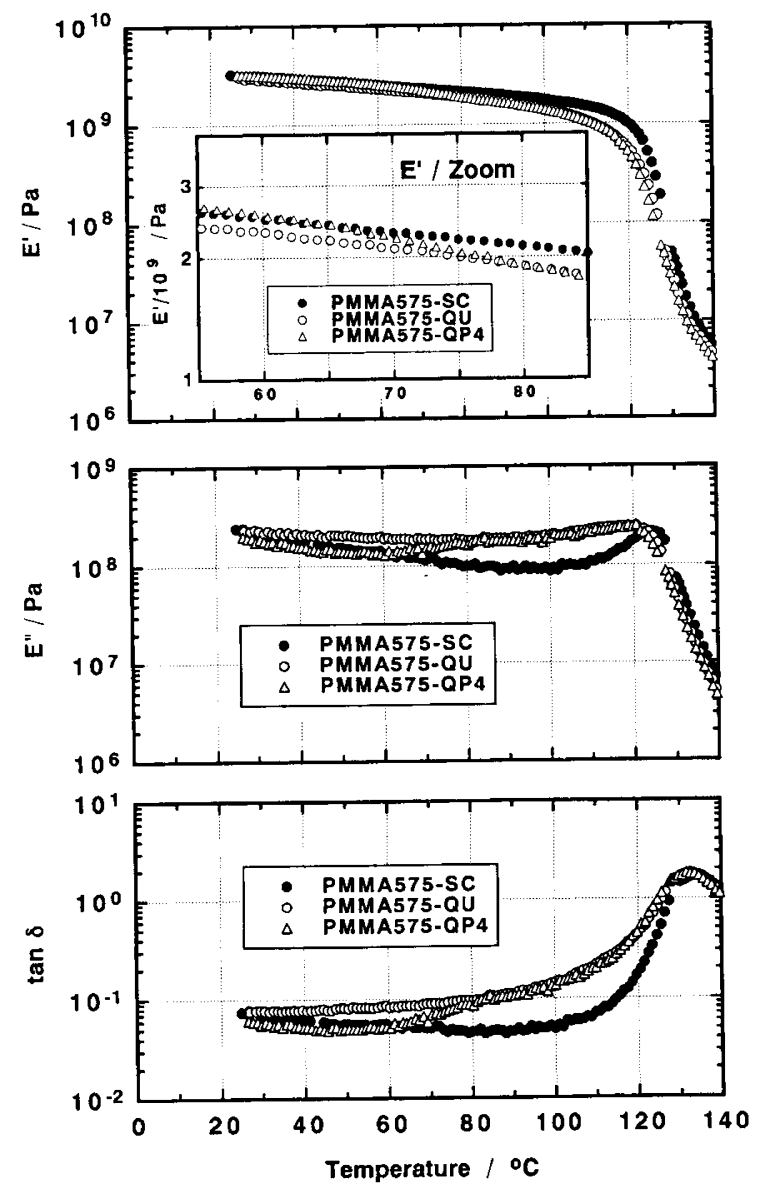

Figure 1. The temperature dispersion curves of $E^{\prime}, E^{\prime \prime}$, and $\tan \delta$ of PMMA575 with three typical thermal histories ; PMMA575-SC, $-\mathrm{QU}$, and -QP4 which was physically aged for one year after QU.

perature dispersion curves of PMMA575-SC physically aged at room temperature for 365 days were also measured, although the curves are not shown here. The data coincided with those of the SC sample over the whole range of temperature measured, indicating clearly that the viscoelasticity is not affected by aging for the SC samples. PMMA72 also showed the similar behavior to PMMA575 as described above.

Figure 2 is a magnification of the data which are measured in temperature range $0-140^{\circ} \mathrm{C}$ as shown in Figure 1 , to be able to see the details. The curves for other samples (QP1-3) were added. For PMMA575 (Figure $2 \mathrm{a}$ ), the dispersion curves of QP samples form an envelope at high temperatures, which coincides with the dispersion curve of the QU. At low temperatures, however, an envelope is formed by the $\mathrm{QP}$ samples. The curves at low temperatures gradually decrease in value with aging time, and all curves are located in the lower side than the curve of the SC. PMMA72 (Figure 2b) shows the similar behavior, and the difference at low temperatures becomes clearer. As the aging time increases, the crossover curve appearing in the middle temperature range moves to the high temperatures.

Figure 3 shows the tan $\delta$ curves of QA annealed at 80 and $110^{\circ} \mathrm{C}$ for PMMA575 and PMMA72. The figure also contains the curves of SC and QU. The results show a similar tendency to that shown in Figure 2. For both types of PMMA, the curves of QA samples can be divided into two groups. One is the group composed of QA1-QA
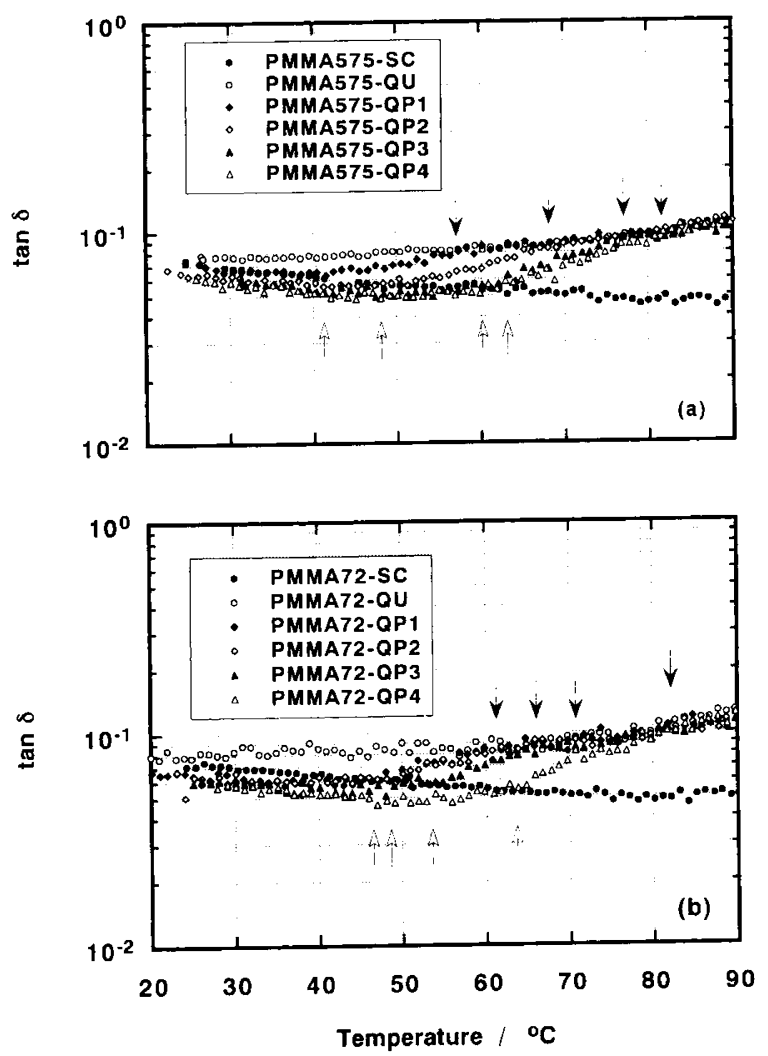

Figure 2. The temperature dispersion curves of $\tan \delta$ of PMMAQP series with PMMA-SC and -QU. (a) PMMA575 and (b) PMMA 72 series. $T_{\mathrm{L}}$ and $T_{\mathrm{H}}$ are pointed out by white and black arrows, respectively.

3 annealed at $80^{\circ} \mathrm{C}$, and the other group composed of QA 4 and QA5 annealed at $110^{\circ} \mathrm{C}$. The two groups are different in temperature range of the crossover. The range is lower for the samples annealed at $80^{\circ} \mathrm{C}$ than for those at $110^{\circ} \mathrm{C}$. Focusing on the curves for the samples at $T_{\mathrm{A}}=80$ ${ }^{\circ} \mathrm{C}$, the crossover temperature moves to the higher side as annealing time increases. This is true for the both types of the polymers. For the samples at $T_{\mathrm{A}}=110^{\circ} \mathrm{C}$, the crossover curve moves in the similar way depending on the annealing time for PMMA575, while the curves are almost unchanged for PMMA72 even if the annealing time is varied. QA samples form an envelope at low temperatures, and the envelope agrees with the SC curve. The other envelope formed by QA samples at high temperatures agrees with the QU curve.

Figure 4 shows the temperature dispersion curves of the viscoelastic functions of QPA samples annealed after physical aging at $T_{\mathrm{r}}$, together with those of QA samples with the same annealing conditions. The $\tan \delta$ curves of PMMA575-QPA1 (closed circles) and PMMA72-QPA1 are slightly different from those of PMMA575-QA1 (open circles) and PMMA72-QA1 at crossover temperatures (Figures 4a and 4c). The curve of PMMA575-QPA5 coincides well with that of PMMA575-QA5 (Figure 4b).

The low temperature portion of the $\tan \delta$ curves of QP samples were located below SC (see Figure 2) curve before annealing at $T_{\mathrm{A}}$, and moved to a similar level of SC curve by annealing at $T_{\mathrm{A}}$ (compare Figures 3 and 4 ). The behavior suggests that memory of physical aging at $T_{\mathrm{r}}$ in the QP samples disappeared by annealing. The memory, however, would remain and appear at crossover tem- 

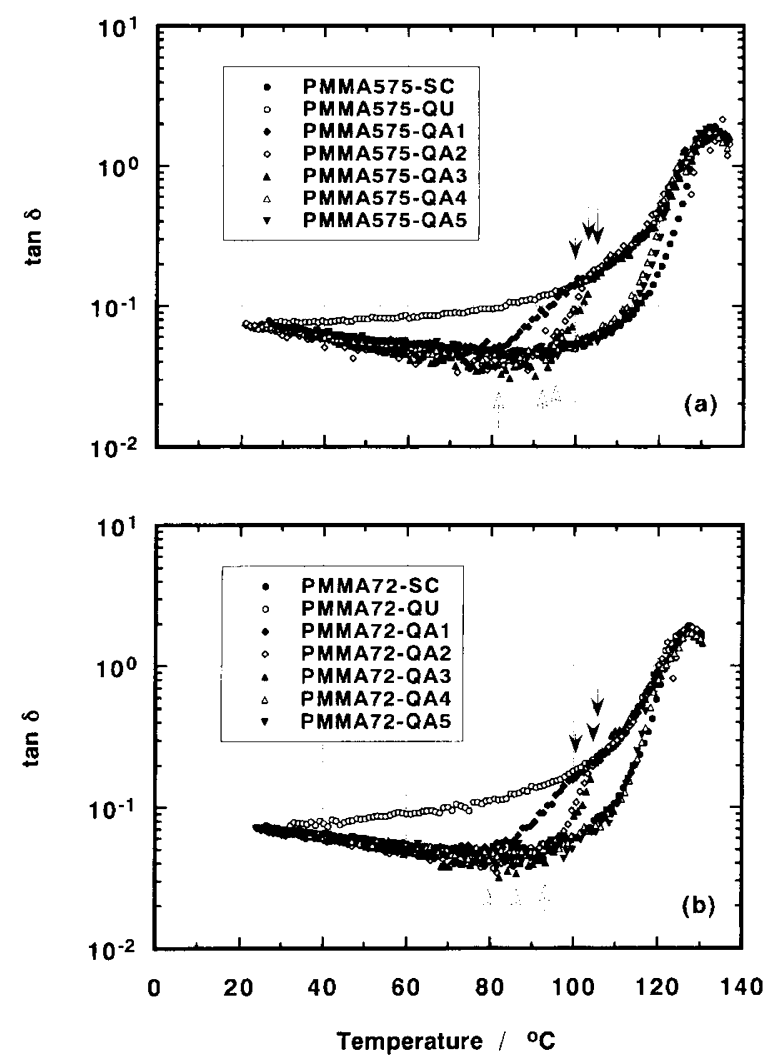

Figure 3. The temperature dispersion curves of $\tan \delta$ of PMMAQA series with PMMA-SC and -QU. The arrows are marked on the curves of samples annealed at $80^{\circ} \mathrm{C}$. (a) PMMA575 and (b) PMMA 72 series. $T_{\mathrm{L}}$ and $T_{\mathrm{H}}$ are pointed out by white and black arrows, respectively.

perature portion of the $\tan \delta$ curves of QPA1 samples. On the other hand, the memory of quenching from $190^{\circ} \mathrm{C}$ remains both in QPA and QA samples, because the envelope in the high temperature region coincides with the curve of QU (see Figure 3).

We can define two characteristic temperatures in Figures 2 and 3 . One is a temperature $\left(T_{\mathrm{L}}\right)$ at which the $\tan \delta$ curves start to be upturned, and the other is a temperature $\left(T_{\mathrm{H}}\right)$ at which the tan $\delta$ curves merge into the envelope at high temperatures. $T_{\mathrm{L}}$ and $T_{\mathrm{H}}$ increase with aging time. Assuming that the size of segmental mobility corresponds to the temperature, we can say that the segmental conformation below $T_{\mathrm{L}}$ has already relaxed and those above $T_{\mathrm{H}}$ has not.

The values of $T_{\mathrm{L}}$ and $T_{\mathrm{H}}$ for PMMA575 and PMMA72 are plotted against aging time in Figure 5. Data points for $T_{\mathrm{L}}$ and $T_{\mathrm{H}}$ can be well represented by a single line, as can be seen from the figure. The effects of molecular weight on the characteristic times are not observed at $T_{\mathrm{A}}$ $=T_{\mathrm{r}}$ and $T_{\mathrm{A}}=80^{\circ} \mathrm{C}$. Annealing at high temperature accelerates the effect of aging on $T_{\mathrm{L}}$ and $T_{\mathrm{H}}$. It is well known that the rate of change of viscoelastic properties depends on the annealing temperature, and the elevation of annealing temperature enhances the rate monotonously. ${ }^{18}$

$T_{\mathrm{L}}$ of QA samples is not directly used when we discuss the results of aging at different temperatures, because $T_{\mathrm{L}}$ depends on the details of the process, and the shape of temperature dispersion curves of viscoelastic functions of PMMA varies by cooling process. ${ }^{17,21}$ On the
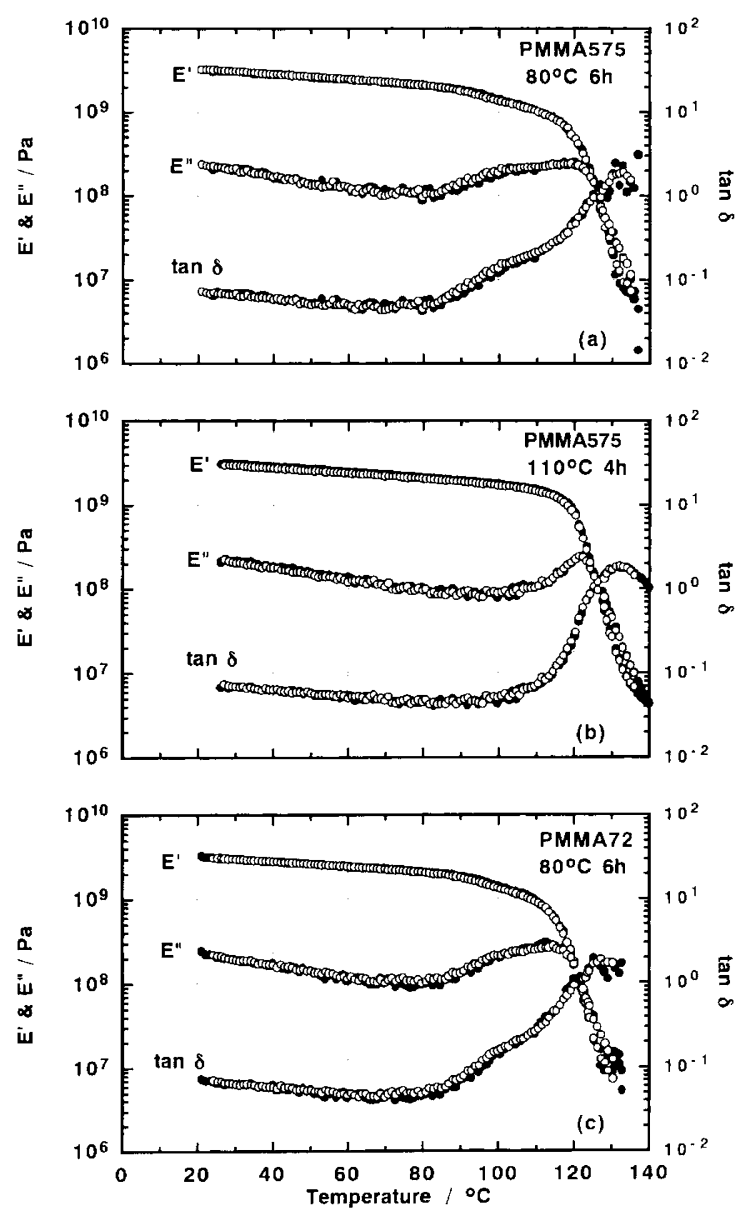

Figure 4. The temperature dispersion curves of $E^{\prime}, E^{\prime \prime}$, and $\tan \delta$ of QPA and QA series. (a) PMMA575-QPA1 and -QA1, (b) PMMA 575-QPA5 and -QA5, and (c) PMMA72-QPA1 and -QA1. QPA and $(\bigcirc) \mathrm{QA}$.

other hand, $T_{\mathrm{H}}$ might be characterized as the temperature corresponding to the segmental conformational size, above which has not been relaxed. When samples come to the equilibrium state, $T_{\mathrm{H}}$ would show no more changes. $T_{\mathrm{H}}$ would be slightly higher than the peak temperature of $\tan \delta$ curve of SC, because the SC process did not attain the real equilibrium as discussed later. From Figure 5 we estimate the time when QU sample comes to the equilibrium state by aging at $T_{\mathrm{r}}$ and $80^{\circ} \mathrm{C}$ respectively by assuming $T_{\mathrm{H}}$ at the equilibrium. For $T_{\mathrm{H}}=130$ ${ }^{\circ} \mathrm{C}$, the time estimated is $1.8 \times 10^{9} \mathrm{~h}$ at $T_{\mathrm{r}}$ and $3.6 \times 10^{6}$ $\mathrm{h}$ at $80^{\circ} \mathrm{C}$ for PMMA72. The rate of change at $80^{\circ} \mathrm{C}$ is much faster than that of at $T_{\mathrm{r}}$.

As stated previously, the difference in shape of the dispersion curves of viscoelastic functions of $\mathrm{QU}$ samples by aging and annealing appeared clearly at lower temperatures, and the effect of aging and annealing extends to higher temperatures with increasing aging and annealing time, but the change did not appear at high temperatures. The rate of change was faster when annealing temperature was high. The difference is caused by the rate of segmental motion in sample. Mobility of segments in main chain is dominated by temperature, and increases with temperature. The movable segment length also increases with temperature. We regard the movable largest segment as a characteristic length at a temperature. The characteristic length is smaller than 

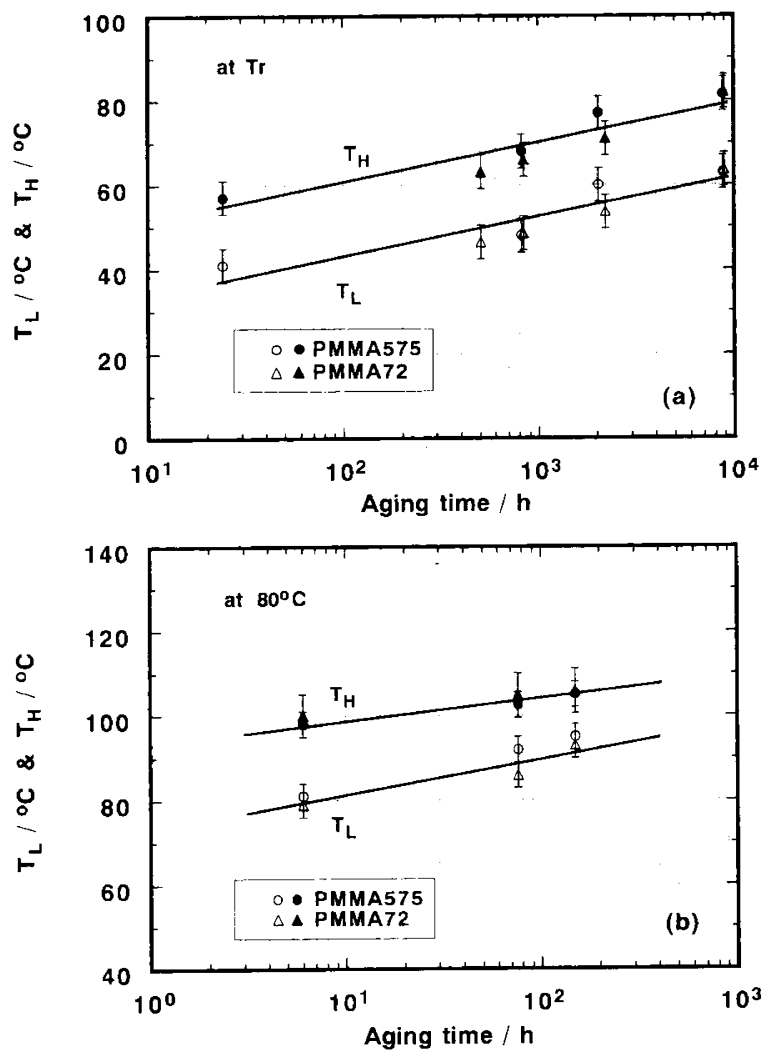

Figure 5. Semi-logarithmic plot of aging time $v s . T_{\mathrm{L}}$ and $T_{\mathrm{H}}$. These were obtained from the results of physical aging at (a) $T_{\mathrm{r}}$ and (b) $80^{\circ} \mathrm{C}$ for PMMA 575 and PMMA 72.

the full length of main chain below $T_{\mathrm{g}}$. The change in shape on the curves proceeding to higher temperatures must correspond to development of conformational relaxation from smaller to larger characteristic length scale undergone at the aging and annealing temperatures.

Figure 6 shows the tan $\delta$ curves of PMMA-SC, -QU, and -QA2 measured at frequency of $100 \mathrm{~s}^{-1}$ for PMMA 575 and PMMA72. The behavior is consistent with that in Figure 3 which were measured at $1 \mathrm{~s}^{-1}$. Thus the change of temperature dispersion of viscoelasticity of PMMA by aging and annealing is not a specific phenomenon at $\omega=1 \mathrm{~s}^{-1}$ but a general one at any frequency. The very broad $\beta$ transition appears at around $60^{\circ} \mathrm{C}$ in Figure 6, that was not observed at $\omega=1 \mathrm{~s}^{-1}$ (Figure 3). The $\beta$ transition of QA2 is seen clearly and that of QU is not clear as shown in the figure. According to literatures the temperature range in which the aging affects the thermal properties of polymeric glasses from $T_{\beta}$ to $T_{\mathrm{g}}{ }^{17,24}$ or from temperatures well below $T_{\beta}$ to $T_{\mathrm{g}}{ }^{18,20}$

\section{Density of PMMA Samples}

Densities of PMMA72 samples are shown in Table II. Densification occurred during physical aging at room temperature. Density of PMMA72-QP4 (1.1988) is higher than that of PMMA72-QU (1.1968), and is also higher than that of PMMA72-SC (1.1985). This suggests that PMMA72-SC is not in the equilibrium state. Since the temperature dispersion curve for PMMA72-QP4 in Figure 2 indicates that the sample is not yet equilibrated, density of QP sample would still increase as the aging time increases further. Since the density of QP4 is
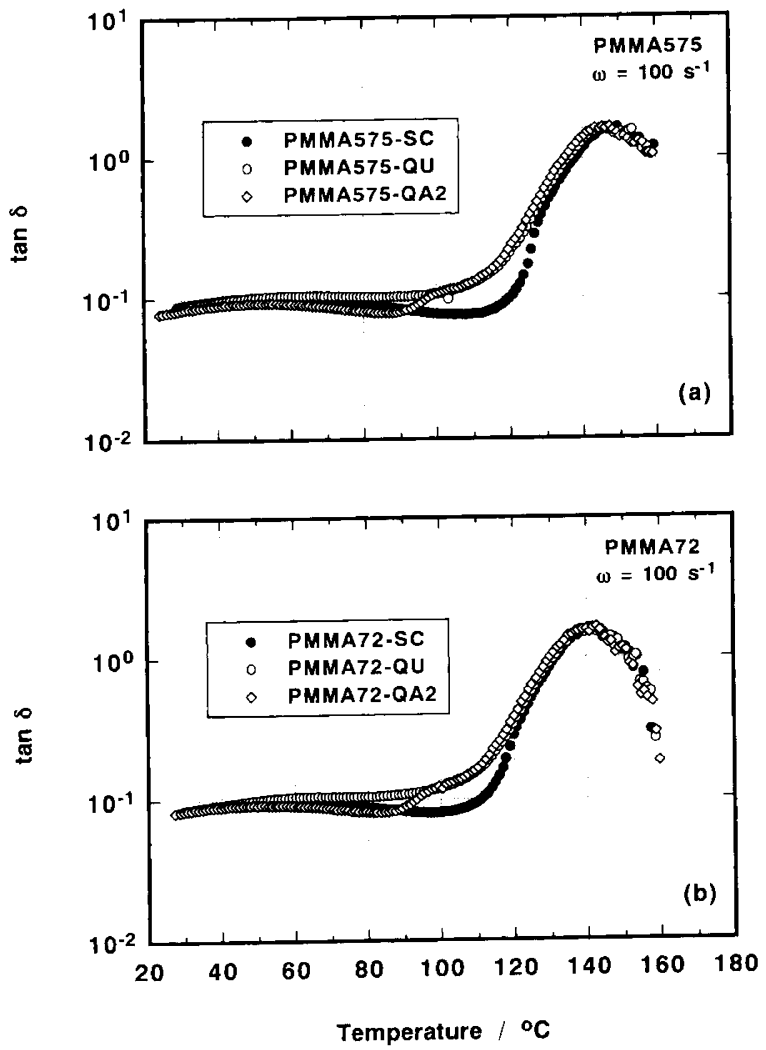

Figure 6. The temperature dispersion curves of $\tan \delta$ of PMMASC, -QU, and -QA 2 measured under $100 \mathrm{~s}^{-1}$ in angular frequency. (a) PMMA 575 and (b) PMMA 72 series.

higher than that of $\mathrm{SC}$, it may be possible to prepare a QP sample with the same density as SC by adjusting the aging time. Even in this case, the QP sample must show the viscoelasticity characteristic of the quenched and then physical aged sample, QP1-4 ; namely, the crossover exists on the dispersion curve of $\tan \delta$. This suggests that the viscoelastic properties cannot be determined only by the density of sample.

The densities of PMMA72-QA1 (1.1993) and -QA2 (1.1994) annealed at $80^{\circ} \mathrm{C}$ are very close to each other, and the situation is the same in density of PMMA72-QA $4(1.1990)$ and -QA5 (1.1990) annealed at $110^{\circ} \mathrm{C}$. The values for the QA samples are higher than that of PMMA 72-SC. Although the temperature dispersion curves of tan 8 of PMMA72-QA4 and -QA5 are almost identical to that of PMMA72-SC (Figure 3), the values of density of the QA samples are higher than that of SC. This suggests that PMMA72-QA4 and -QA5 are closer to the equilibrium than PMMA72-SC.

Polymeric glass becomes dense with relaxation. QA1-2 samples have higher density than QA4-5 samples. The former seems to be more relaxed than the latter, but this is not true as shown in Figure 3. We must consider the thermal expansion of sample. Density of polymeric glass is low with rising temperature. The experimental results of density are reasonable when QA1-2 and QA4-5 samples were fixed near the equilibrium values of densities at 80 and $110^{\circ} \mathrm{C}$. It should be noted that degree of relaxation of sample is not judged by the only density value at $30^{\circ} \mathrm{C}$.

Density of PMMA72-QPA1 (1.1997) is higher than that of PMMA72-QA1 (1.1993), density of PMMA72- 
QPA5 (1.1989) is very close to that of PMMA72-QA5 (1.1990). QPA1 has maximum value in this work. The sample experienced two thermal histories ; physical aging at $T_{\mathrm{r}}$ and annealing at $80^{\circ} \mathrm{C}$. The thermal histories made samples dense as stated before. The agreement of QPA5 and QA5 suggests that annealing at $110^{\circ} \mathrm{C}$ is effective and eliminates the memory of thermal history at $T_{\mathrm{r}}$ in QPA5. The eliminating memory would be also suggested by the coincidence of $\tan \delta$ curves between QPA5 and QA5 of PMMA575 (Figure 4b). On the other hand, the result of QPA1 is complicated. The result suggests that densification occurs by two thermal histories. The memory of thermal history at $T_{\mathrm{r}}$ lessened but remains in QPA1 samples. This would be corresponding to the result of viscoelasticity at crossover temperature region of $\tan \delta$ curves of PMMA575-QPA1 and PMMA72-QPA1 (Figures $4 \mathrm{a}$ and $4 \mathrm{c}$ ).

The changes in viscoelasticity and density of QU sample are due to the conformational relaxation, and both must be related to each other. We can assume that QU sample consists of low-density and high-density regions at the initial stage of aging and annealing. The region grows gradually during aging, and the change is according to the conformational relaxation from smaller to larger length scale of segment at a temperature. Lowdensity region in the sample keeps the memory of quenching.

A well known 'memory effect' has been investigated by volumetric recovery of polymeric glass. ${ }^{4,5}$ The effect appears when a sample has been treated at three near temperatures $\left(T_{1}>T_{2}>T_{3}\right)$. The sample was quenched from $T_{1}$ to $T_{3}$ and kept at $T_{3}$ for a while, and then the sample heated up to $T_{2}$. Volumetric change of the sample shows a maximum during aging at $T_{2}$, because the sample has structures formed at $T_{1}$ and $T_{3}$. We consider the memory effect on the viscoelastic properties of QPA samples. QPA samples kept the memory at $T_{1}\left(=190^{\circ} \mathrm{C}\right)$ that were shown by the coincidence in $\tan \delta$ curves of QPA and QU samples at high temperatures. On the other hand, the memory at $T_{3}$ (= room temperature) disappeared or lessened from QPA sample. This would occur by large temperature jump from room temperature to 80 or $110^{\circ} \mathrm{C}$. Thus the idea of memory effect would be valid for the change of temperature dispersion of viscoelastic properties by aging.

\section{CONCLUSIONS}

The conformational and volumetric relaxation of PMMA in the glassy state was investigated by dynamic viscoelasticity and density measurements. Change of dispersion curves of viscoelastic functions of $Q U$ samples by aging and annealing appears clearly at lower temperatures, proceeding to higher temperatures with aging and annealing time, but does not appear at higher tem- peratures. The rate of change becomes fast as annealing temperature becomes high, but is independent of the molecular weight. Densification occurs for QU samples by the physical aging at room temperature, and annealing at 80 and $110^{\circ} \mathrm{C}$.

The changes of viscoelasticity and density of QU sample by aging and annealing are due to conformational relaxation. The relaxation is expected to extend from smaller to larger length scale of segment with time. Densification is also related to the relaxation.

Acknowledgment. This study was supported by Grant-in-Aid for Scientific Research (Nos. 10305069 and 10450367) from the Ministry of Education, Science, Sports and Culture of Japan, and by JSPS Research for the Future Program, Biological Tissue Engineering Project, No. JSPS-RFTF 98100201.

\section{REFERENCES}

1. L. C. E. Struik, "Physical Aging in Amorphous Polymers and Other Materials", Elsevier, Amsterdam, (1978).

2. I. M. Hodge, J. Non-Cryst. Solids, 169, 211 (1994).

3. J. M. Hutchinson, Prog. Polym. Sci., 20, 705 (1995).

4. A. J. Kovacs, J. J. Aklonis J. M. Hutchinson, and A. R. Ramos, J. Polym. Sci., Polym. Physics Ed., 17, 1097 (1979).

5. K. Adachi and T. Kotaka, Polym. J., 14, 959 (1982).

6. J. Perez, J. Y. Cavaille, R. D. Calleja, and J. L. G. Ribelles, Makromol. Chem., 192, 2141 (1991).

7. G. B. McKenna, Y. Leterrier, and C. R. Schultheisz, Polym. Eng. Sci., 35, 403 (1995).

8. S. L. Simon, D. J. Plazek, J. W. Sobieski, and E. T. McGregor, J. Polym. Sci., Polym. Physics, 35, 929 (1997).

9. L. C. E. Struik, Polymer, 38, 4053 (1997).

10. S. Montserrat, J. L. Gomez-Ribelles, and J. M. Meseguer, Polymer, 39, 3801 (1998).

11. S. L. Simon and G. B. McKenna, J. Chemical Physics, 107, 8678 (1997).

12. I. Echeverria, P-C, Su, S. L. Simon, and D. J. Plazek, J. Polym. Sci., Polym. Physics, 35, 929 (1997).

13. T. Ricco and T. L. Smith, Polymer, 26, 1979 (1985).

14. J. M. G. Cowie, R. Ferguson, S. Harris, and J. McEwen, Polymer, 39, 4397 (1998).

15. P. A. O'Connell and G. B. McKenna, Polym. Eng. Sci., 37, 1785 (1997).

16. L. Guerdoux, R. A. Duckett, and D. Froelich, Polymer, 25, 1392 (1984).

17. L. C. E. Struik, Polymer, 28, 57 (1987).

18. R. Diaz-Calleja, A. Ribes-Greus, and J. L. Gomez-Ribelles, Polymer, 30, 1433 (1989).

19. O. Araki, Q. Zheng, M. Takahashi, T. Takigawa, and T. Masuda, Matr. Sci. Res. Int., 1, 144 (1995).

20. R. A. Venditti and J. K. Gillham, J. Appl. Polym. Sci., 45, 501 (1992).

21. O. Araki, T. Yoshizawa, and T. Masuda, Polym. Bull., 42, 205 (1999).

22. M. Alcoutlabi and J. J. Martinez-Vega, Polymer, 39, 6269 (1998).

23. A. Faivre, L. David, R. Vassoille, G. Vigier, S. Etienne, and E. Geissler, Macromolecules, 29, 8387 (1996).

24. A. B. Brennan and F. Feller III, J. Rheol., 39, 453 (1995). 